\title{
Useful transformations between photometric systems
}

\author{
P. Harmanec ${ }^{1,2}$ and H. Božićí ${ }^{2,3}$ \\ 1 Astronomical Institute of the Charles University, V Holešovičkách 2, 18000 Praha 8, Czech Republic \\ 2 Astronomical Institute, Academy of Sciences, 25165 Ondřejov, Czech Republic \\ 3 Hvar Observatory, Faculty of Geodesy, Zagreb University, Kačićeva 26, 10000 Zagreb, Croatia \\ e-mail: hbozic@geodet.geof.hr
}

Received 15 August 2000 / Accepted 31 January 2001

\begin{abstract}
In the course of detailed studies of the long-term variations of particular Be stars, we have practically verified that the non-linear transformations, needed for a reliable transformation between a standard and an instrumental photometric system can also be successfully used to the transformations between two particular standard photometric systems. To demonstrate the importance and power of non-linear transformations and to provide pieces of information useful for a broader astronomical community, we summarize the transformation formulæ already published (but hidden from the general community in papers dealing with particular Be stars) and present several other useful transformations derived by us. All transformation formulæ presented here are transformations from various photometric systems to Johnson's $U B V$ system. However, the general procedures applied here would allow construction of similar transformations between other photometric systems, too.
\end{abstract}

Key words. methods: observational - techniques: photometric

\section{Transformations to a standard system}

Prime reductions of photoelectric observations, i.e. transformations of observed instrumental magnitudes to instrumental magnitudes outside the Earth atmosphere, and transformations of instrumental magnitudes to a standard system, are considered well-established procedures. The principal steps of these reductions were described in the influential paper by Hardie (1962) and many photometrists still continue to follow his advice. This is unfortunate since it was shown several times cf., e.g. Cousins \& Jones (1976), Harmanec et al. (1994), Young (1992) among others - that the linear transformation formulæ given by Hardie (1962) do not ensure accurate enough reductions. The most comprehensive accounts of why the very nature of the problem dictates the use of non-linear tranformation formulæ even for narrowband photometric systems were given by Young (1992) and Harmanec et al. (1994) where also references to previous work can be found. The studies that followed had demonstrated that the consistent application of nonlinear transformation formulæ and determination of accurate $U B V$ magnitudes of many non-variable stars by Harmanec et al. (1994) led in a sense to a "rehabilitation" of the $U B V$ system since all three $U B V$ magnitudes can now be derived from different stations and instrumen-

Send offprint requests to: P. Harmanec,

e-mail: hec@sunstel.asu.cas.cz tations (even from stations near the sea level and highmountain stations) with an accuracy of about $0^{\mathrm{m}} \cdot 01$ from all-sky observations. This was convincingly demonstrated by Harmanec (1998). He was able to define a very accurate transformation between the broad-band Hipparcos $H_{\mathrm{p}}$ magnitude published by Perryman et al. (1997) and mean $U B V$ magnitudes from Hvar and Skalnaté Pleso derived by Harmanec et al. (1994). Such an accurate transformation would not be possible if any of the two data sets were not reduced properly.

\section{Transformations between two existing systems}

For convenience of the astronomical community we summarize here several useful transformation formulæ which allow reliable transformations of various photometries to passbands of the standard Johnson system.

First, we give the transformations between two wellestablished photometric systems:

In their study of the Be binary V360 Lac, Hill et al. (1997) derived the following transformation from the $D A O$ system - see, e.g., Hill et al. (1976) - to the $U B V$ system:

$$
\begin{aligned}
V= & {[55] } \\
(B-V)= & 1.1348 X+0.02368 Y \\
(U-B)= & 0.24453 X+0.74611 Y \\
& -0.37301 X^{2}+0.50754 X^{3}
\end{aligned}
$$


where $X=[44]-[55]$ a $Y=[35]-[44]$.

In a study of the Be star OT Gem, Božić et al. (1999) published a transformation between the 13-colour photometry by Johnson \& Mitchell (1975) and the $U B V$ system but gave incomplete information on the meaning of the symbols used. Here, we reproduce this transformation in the proper, directly usable form:

$$
\begin{aligned}
V= & m_{55}+0.01930 b v+0.01830 u b \\
& -0.06538 b v^{2}+0.02411 b v^{3}+0.01434 \\
B= & m_{43}-0.03528 b v+0.01464 u b \\
& -0.02837 b v^{2}-0.03429 b v^{3}+0.00006 \\
U= & m_{35}+0.10478 b v-0.15289 u b \\
& +0.11294 b v^{2}-0.06538 b v^{3}+0.01686,
\end{aligned}
$$

where

$$
\begin{aligned}
m_{35} & =m_{52}+[(33-52)+(35-52)+(37-52)] / 3, \\
m_{43} & =m_{52}+[(45-52)+(40-52)] / 2, \\
m_{55} & =m_{52}+(52-58) / 2 \\
b v & =m_{43}-m_{55} \\
u b & =m_{35}-m_{43} .
\end{aligned}
$$

The symbols $m_{52},(33-52)$, etc. denote the reference magnitudes and colour indices of the Johnson 13-C system.

For the first time we present here a transformation from the Geneva 7-colour photometry to the Johnson system. To obtain it, we used, as in other cases, all constant stars with good $U B V$ values from Harmanec et al. (1994) for which we could also find Geneva photometry in the compilation by Burki et al. (1998). It turned out that a bilinear transformation is sufficient for the $V$ magnitude and the transformation reads as follows:

$$
\begin{aligned}
V= & V_{\mathrm{G}}+0.02161 B V+0.01209 U B-0.01658 \\
B= & B_{\mathrm{G}}+0.21633 B V-0.00252 U B \\
& -0.01643 B V^{2}+0.00120 B V^{3}+0.78412, \\
U= & U_{\mathrm{G}}-0.18411 B V-0.26145 U B \\
& +0.01763 B V^{2}+0.06330 B V^{3}-0.02095,
\end{aligned}
$$

where $B V=(V-B)_{\mathrm{G}}$ and $U B=(U-B)_{\mathrm{G}}$ (i.e. colours $B$ and $U$ in the usual Geneva group tabulation). The rms errors of the fits per 1 observation amount to 0.0102 , 0 . 0100 , and 0.0254 for $V, B$ and $U$, respectively.

Finally, we also derived new transformations between Strömgren and Johnson colours, using the weighted mean Strömgren colours from the catalogue of Hauck \& Mermilliod (1998). They read as follows:

$$
\begin{aligned}
(B-V)= & 1.41694 b v+0.07010 u b \\
& +0.57145 b v^{2}-0.60399 b v^{3}-0.10118, \\
(U-B)= & 0.66567 u b-0.09718 b v \\
& +0.24407 b v^{2}+0.29340 b v^{3}-0.91958,
\end{aligned}
$$

where $b v=(b-y)$ and $u b=(u-b)$ are the Strömgren colours. The rms errors of the fits per 1 observation are 0 . 0170 and 0.0165 for the $B-V$ and $U-B$, respectively.
Since in many catalogues, values $V,(b-y), m_{1}$ and $c_{1}$ are tabulated for Strömgren photometry, we remind that

$$
\begin{aligned}
b & =V+(b-y) \\
v & =b+(b-y)+m_{1}=V+2(b-y)+m_{1}, \\
u & =v+(b-y)+m_{1}+c_{1} \\
& =V+3(b-y)+2 m_{1}+c_{1}, \\
(u-b) & =2(b-y)+2 m_{1}+c_{1}
\end{aligned}
$$

It is interesting to compare our transformation to that published by Turner (1990). He derived his transformation formulæ in the form

$$
(B-V)=1.584(b-y)+0.681 m_{1}-0.116,
$$

$(U-B)=0.675(u-b)-0.938$,

valid only for hot stars with $(u-b) \leq 2.0$. He obtained rms errors of 0.016 and 0.022 for $B-V$ and $U-B$, respectively. As a matter of curiosity, we reproduced his second relation, also for hot stars only, in the form

$(U-B)=0.659(u-b)-0.912$,

the rms error per 1 observation being $0{ }^{\mathrm{m}} 0174$ only. Note that our relations (20) and (21) hold over the whole range of stellar colours.

Since the $(B-V)$ and $(U-B)$ colour indices are often known for the stars in question, it is possible to define transformations to either $V$ or $B$ Johnson magnitude from some photometries based on observations in one passband only.

Investigating $\varphi$ Per, Božić et al. (1995) found that the magnitude differences based on the old photoelectric observations with a Rb diode, which were secured by Guthnick \& Prager (1918), can be reliably transformed into Johnson's $B$ magnitude differences with the help of the following relation:

$\triangle B=\triangle b+0.2366 \triangle(B-V)$.

A similar transformation for early photoelectric photometry from the Lick Observatory, secured by Stebbins (1916) and Stebbins (1921), was derived by Holmgren et al. (1999):

$$
\triangle V=\triangle m_{500}-0.64915 \triangle(B-V)-0.01603 \triangle(U-B) .
$$

Recently, Harmanec (1998) published the following useful transformation which allows an accurate determination of the Johnson $V$ magnitude from the broad-band $H_{\mathrm{p}}$ magnitude from the Hipparcos satellite:

$$
\begin{aligned}
V= & H_{\mathrm{p}}-0.2964(B-V)+0.0050(U-B) \\
& +0.1110(B-V)^{2}+0.0157(B-V)^{3}+0.0072 .
\end{aligned}
$$

A package of FORTRAN programs which allow reliable photometric reductions has been published with detailed documentation by Harmanec \& Horn (1998) and is also available on request from the first author of this note. 
Acknowledgements. We gratefully acknowledge useful comments on the first version of this note provided by Drs. D. H. Holmgren, J. R. Percy, E. Poretti, R. R. Shobbrook, C. Sterken and G. A. H. Walker and by an anonymous referee. This study was supported by grant 30304 of the Granting Agency of the Czechoslovak Academy of Sciences and its finishing was made possible through the support via research plan J13/98: 113200004 Investigation of the Earth and the Universe.

\section{References}

Božić, H., Ruždjak, D., \& Sudar, D. 1999, A\&A, 350, 566

Božić, H., Harmanec, P., Horn, J., et al. 1995, A\&A, 304, 235

Burki, G., et al. 1998, http://obswww. unige.ch/gcpd/cgi-bin/photoSys.cgi

Cousins, A. W. J., \& Jones, D. H. P. 1976, Mem. R. Astron. Soc., 81,1

Guthnick, P., \& Prager, R. 1918, Veröff. Berlin Babelsberg 2, 3,113

Hardie, R. H. 1962, in Stars and Stellar Systems, vol. II: Astronomical Techniques, ed. G. P. Kuiper, \& B. M. Middlehurst (Univ. of Chicago Press, Chicago, USA), 178
Harmanec, P. 1998, A\&A, 335, 173

Harmanec, P., \& Horn, J. 1998, Journal Astron. Data No. 4 CD-ROM file 5

Harmanec, P., Horn, J., \& Juza, K. 1994, A\&AS, 104, 121

Hauck, B., \& Mermilliod, M. 1998, A\&AS, 129, 431

Hill, G., Hilditch, R. W., \& Pfannenschmidt, E. L. 1976, Publ. Dom. Astrophys. Obs., 15, 1

Hill, G., Harmanec, P., Pavlovski, K., et al. 1997, A\&A, 324, 965

Holmgren, D. E., Hadrava, P., Harmanec, P., et al. 1999, A\&A, 345,855

Johnson, H. L., \& Mitchell, R. I. 1975, Rev. Mex. Astron. Astrofís., 1, 299

Perryman, M. A. C, Høg, E., Kovalevsky, J., Lindegren, L., \& Turon, C. 1997, ESA SP-1200, The Hipparcos and Tycho Catalogues

Stebbins, J. 1916, Lick Obs. Bull., 8, No. 277, 186 \& 192

Stebbins, J. 1921, ApJ, 54, 81

Turner, D. G. 1990, PASP, 102, 1331

Young, A. T. 1992, A\&A, 257, 366 